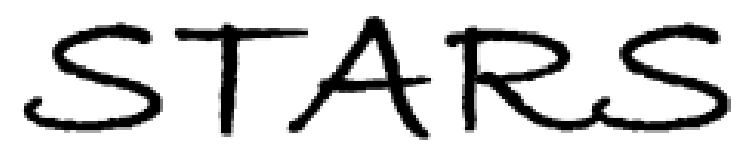

University of Central Florida

STARS

$1-1-2012$

\title{
Electron mean free path of tungsten and the electrical resistivity of epitaxial (110) tungsten films
}

Dooho Choi

Chang Soo Kim

Doron Naveh

Suk Chung

Andrew P. Warren

University of Central Florida

See next page for additional authors

Find similar works at: https://stars.library.ucf.edu/facultybib2010

University of Central Florida Libraries http://library.ucf.edu

This Article is brought to you for free and open access by the Faculty Bibliography at STARS. It has been accepted for inclusion in Faculty Bibliography 2010 s by an authorized administrator of STARS. For more information, please contactSTARS@ucf.edu.

\section{Recommended Citation}

Choi, Dooho; Kim, Chang Soo; Naveh, Doron; Chung, Suk; Warren, Andrew P.; Nuhfer, Noel T.; Toney, Michael F.; Coffey, Kevin R.; and Barmak, Katayun, "Electron mean free path of tungsten and the electrical resistivity of epitaxial (110) tungsten films" (2012). Faculty Bibliography 2010s. 2414.

https://stars.library.ucf.edu/facultybib2010/2414

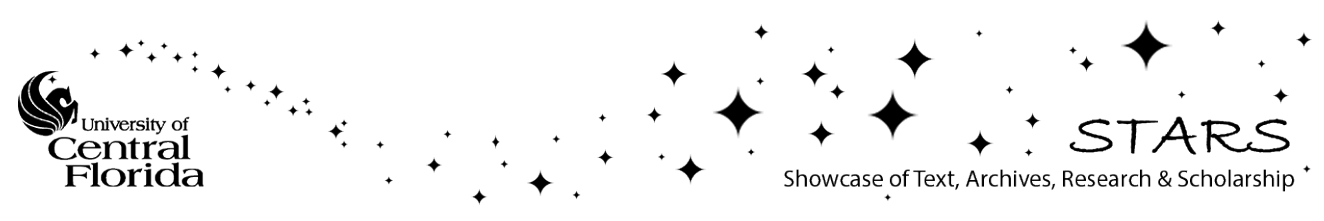




\section{Authors}

Dooho Choi, Chang Soo Kim, Doron Naveh, Suk Chung, Andrew P. Warren, Noel T. Nuhfer, Michael F. Toney, Kevin R. Coffey, and Katayun Barmak 


\title{
Electron mean free path of tungsten and the electrical resistivity of epitaxial (110) tungsten films
}

\author{
Dooho Choi, ${ }^{1}$ Chang Soo Kim, ${ }^{2}$ Doron Naveh, ${ }^{2}$ Suk Chung, ${ }^{1}$ Andrew P. Warren, ${ }^{3}$ Noel T. Nuhfer, ${ }^{1}$ Michael F. Toney, ${ }^{4}$ \\ Kevin R. Coffey, ${ }^{3}$ and Katayun Barmak ${ }^{1}$ \\ ${ }^{1}$ Department of Materials Science and Engineering, Carnegie Mellon University, 5000 Forbes Avenue, Pittsburgh, Pennsylvania, 15213, USA \\ ${ }^{2}$ Department of Electrical and Computer Engineering, Carnegie Mellon University, 5000 Forbes Avenue, Pittsburgh, \\ Pennsylvania 15213, USA \\ ${ }^{3}$ Advanced Materials Processing and Analysis Center, University of Central Florida, 4000 Central Florida Boulevard, \\ Orlando, Florida 32816, USA \\ ${ }^{4}$ Stanford Synchrotron Radiation Lightsource, 2575 Sand Hill Road, Menlo Park, California 94025, USA \\ (Received 18 November 2011; revised manuscript received 12 June 2012; published 23 July 2012)
}

\begin{abstract}
This work describes a study of the classical electrical resistivity size effect in tungsten. The important length scale for this size effect is the isotropic average electron mean free path (EMFP), which was determined to be $19.1 \mathrm{~nm}$ for $\mathrm{W}$ at $293 \mathrm{~K}$ by employing density functional theory. To explore the size effect experimentally, (110) oriented epitaxial W films with thicknesses ranging from 9.8 to $299.7 \mathrm{~nm}$ were prepared by sputter deposition onto (11) 0 ) $\mathrm{Al}_{2} \mathrm{O}_{3}$ substrates at $520{ }^{\circ} \mathrm{C}$ followed by postdeposition annealing in $\mathrm{Ar}-4 \% \mathrm{H}_{2}$ at $850{ }^{\circ} \mathrm{C}$. Film resistivities were measured at room temperature and at liquid He temperature. The Fuchs-Sondheimer (FS) surface scattering model with a low specularity parameter $(p=0.11)$ was shown to provide a good description of the film resistivity as a function of film thickness. Further, it is shown that an upper bound to the EMFP cannot be established by fitting resistivity data to the FS model, whereas a lower bound can be assessed.
\end{abstract}

DOI: 10.1103/PhysRevB.86.045432

PACS number(s): 85.40.Ls, 81.15.Kk, 81.15.Cd, 71.20.Be

\section{INTRODUCTION}

Tungsten has recently been gaining attention as a potential replacement for $\mathrm{Cu}$ in semiconductor metallization because of its very high melting point $(3695 \mathrm{~K}$ compared with $\mathrm{Cu}$ at $1357 \mathrm{~K}$ ), which should translate to greater reliability due to higher activation barriers for diffusion in the grain boundaries and along surfaces. ${ }^{1-4}$ However, with regard to the resistivity size effect, i.e., whether $\mathrm{W}$ can exhibit a lower resistivity than $\mathrm{Cu}$ at small dimensions despite its higher bulk resistivity $(5.3 \mu \Omega \mathrm{cm}$ compared to $\mathrm{Cu}$ at $1.7 \mu \Omega \mathrm{cm})$, it is necessary to quantify the relative contributions of the classical resistivity size effect scattering mechanisms to the increased resistivity with decreasing size (line height, linewidth, and grain size). ${ }^{5}$ Two of the most important of these mechanisms are surface scattering and grain boundary scattering. 6

Similar to studies of $\mathrm{Cu}$, studies of W (Refs. 7-10) have used the surface scattering model of Fuchs and Sondheimer ${ }^{11,12}$ (FS) alone or in combination with the grain boundary scattering model of Mayadas and Shatzkes ${ }^{13}$ (MS) in interpreting the results. The FS and MS models each incorporate a phenomenological parameter related to electron scattering at defects. For the FS model this parameter is $p$, the fraction of electrons that are specularly scattered from the surfaces. The fraction of electrons diffusely scattered from the surfaces is then $(1-p)$. The film resistivity due to the surface scattering is given by ${ }^{11,12}$

$$
\begin{aligned}
\frac{\rho_{\mathrm{FS}}}{\rho_{o}}= & {\left[1-\left(\frac{3}{2 k}\right)(1-p) \int_{1}^{\infty}\right.} \\
& \left.\times\left(\frac{1}{t^{3}}-\frac{1}{t^{5}}\right) \frac{1-\exp (-k t)}{1-p \exp (-k t)} d t\right]^{-1},
\end{aligned}
$$

where $k=h$ (thickness) $/ \lambda_{0}$ (bulkEMFP) and $\rho_{o}$ is the thick film resistivity of the metal in the limit of infinite thickness and thus includes both the phonon and the impurity scattering contributions. For the MS model, the phenomenological parameter is $R$, the fraction of electrons that are reflected from a grain boundary, with $(1-R)$ denoting the fraction transmitted. $^{13}$

Typically, the parameters $p$ and $R$ are treated as fitting parameters and extracted from the experimental resistivity data. Table I lists these parameters for a representative set of studies of $\mathrm{W}$ over the past 20 years. The use of the FS and MS models in fitting resistivities of films and lines requires knowledge of the EMFP for phonon scattering in the metal at the temperature of measurement. However, unlike $\mathrm{Cu}$, there is no generally agreed EMFP value in W. Table I lists the values of the EMFP either given in or obtained from other data given in the literature for W. These EMFP values cover a wide range, from 2 to $54 \mathrm{~nm}$, which complicates systematic studies of the resistivity size effect in $\mathrm{W}$ films and lines. A detailed discussion of each of the entries in Table I is given in Ref. 14.

Given the large range of values reported for the EMFP, we turn to density functional theory (DFT) to calculate the Fermi surface area $(S)$. Using this value, we find an isotropic average EMFP for $\mathrm{W}$ at $293 \mathrm{~K}$ of $19.1 \mathrm{~nm}$. We then show that the resistivity of (110) oriented epitaxial $\mathrm{W}$ films deposited on (1120) $\mathrm{Al}_{2} \mathrm{O}_{3}$ substrates can be well described by the FuchsSondheimer (FS) model with this value of EMFP and a surface specularity parameter, $p=0.11$. We also report that fitting resistivity data to the FS model in order to estimate an EMFP is not a reliable method since no upper bound to the EMFP can be established using this approach.

\section{CALCULATIONS AND EXPERIMENTS}

To obtain the Fermi surface area of $\mathrm{W}$, we employed density functional theory (DFT) calculations of bulk W using the experimental lattice parameters of the bcc phase $(3.16 \AA)$. We used the generalized gradient approximation (GGA), ${ }^{15}$ as implemented within the ABINIT $^{16}$ software package, with 
TABLE I. Surface specularity parameter $p$, grain boundary reflectivity $R$, and the electron mean free path (EMFP).

\begin{tabular}{lccc}
\hline \hline Reference & $p$ & $R$ & EMFP at room temperature (nm) \\
\hline Steinhögl et al. (2005) (Ref. 7) & 0.3 & 0.25 & 54 \\
Learn and Foster (1985) (Ref. 8) & 1 & $0,0.38-0.67^{\mathrm{a}}$ & 41 \\
Mikhailov et al. (1996) (Ref. 9) & 0.3 & - & 39.6 \\
Rossnagel et al. (2002) (Ref. 10) & 0 & 0 & $10-12$ \\
Choi et al. (2011) (Ref. 4) & - & - & 2 \\
\hline \hline
\end{tabular}

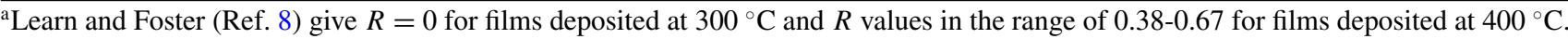

a kinetic energy cutoff of 80 Hartree and a uniform $k$-point mesh of $55 \times 55 \times 55$. Relativistic effects including spin-orbit coupling were accounted for in the calculation by using a relativistic pseudopotential ${ }^{17}$ and by employing the spin-orbit term self-consistently. Isosurface images of the Fermi energy in the Brillouin zone were recorded with the XCRYSDEN software package, ${ }^{18}$ and a numerical integration was conducted to evaluate the corresponding Fermi surface area. ${ }^{19}$

The substrate used for epitaxial growth of $\mathrm{W}$ was $(11 \overline{2} 0)$ $\mathrm{Al}_{2} \mathrm{O}_{3}$. In order to reduce the surface roughness and mosaicity of the substrates, they were annealed at $1000{ }^{\circ} \mathrm{C}$ in air for $2 \mathrm{~h}$ before deposition of the W layer. ${ }^{20,21}$ (110) oriented epitaxial W films with nominal thicknesses ranging from 10 to $300 \mathrm{~nm}$ were deposited at $520{ }^{\circ} \mathrm{C}$ by dc sputtering from a $99.95 \%$ pure $\mathrm{W}$ target. Additional details can be found elsewhere. ${ }^{4}$

The thicknesses of $\mathrm{W}$ films were measured by $\mathrm{x}$-ray reflectivity and transmission electron microscopy (TEM) of cross-sectioned samples. $\theta-2 \theta$ x-ray diffraction (XRD) scans were performed in order to identify phase, epitaxial growth, and the growth orientation. To assess the quality of the epitaxial W films, angular full width at half maximum (FWHM) values from rocking curve ( $\omega$ scan) were measured for the (110) peak. The surface roughness of the $\mathrm{W}$ films was measured by atomic force microscopy (AFM) in the tapping mode.

For microstructural examination, one film, film $\mathrm{F}$, was examined in cross section in the transmission electron microscope (TEM). The sapphire substrate (MTI Corporation) was beveled at two corners so as to clearly mark the side parallel to the $\langle 0001\rangle$ direction. The cross section was prepared perpendicular to this side using a focused ion beam (FIB) system. High-resolution transmission electron micrographs (HRTEM) were recorded at $300 \mathrm{kV}$ using an FEI Titan 80-
300 TEM. The sheet resistance of the films was measured using the van der Pauw method. ${ }^{4,22}$ Resistivities were measured at room temperature and at $4.2 \mathrm{~K}$. Following deposition and after the measurement of film resistivity in the as-deposited state, the films were annealed at $850{ }^{\circ} \mathrm{C}$ for $2 \mathrm{~h}$ in $\mathrm{Ar}-4 \% \mathrm{H}_{2} .{ }^{4}$ The room temperature and $4.2 \mathrm{~K}$ resistivities of the films in the as-deposited state and after the annealing treatment are given in Table II.

\section{RESULTS AND DISCUSSION}

The Fermi surface constructed in the method described earlier is shown in Fig. 1 where the four closed parts of the surface are labeled with their commonly used designations, i.e., electron jack, electron ball, hole octahedron, and hole ellipsoid. The calculated Fermi surface area is $15.1 \AA^{-2}$, in close agreement with the values separately measured by Sparlin and Marcus ${ }^{23}$ and Girvan et al..$^{24}$ using the de Haas-van Alphen (DHVA) effect. However, the value is a factor of 2 greater than the value of $7.0 \AA^{-2}$ reported by Fawcett and Griffiths. ${ }^{25}$

Isotropic average EMFP values over the Fermi surface in metals can be obtained using the relationship between the Fermi surface area $S$ and the dc conductivity $\sigma$ by: $:^{25,26}$

$$
\sigma=\frac{e^{2} S}{6 \pi^{2} h}\left\langle\lambda_{0}\right\rangle,
$$

where $e$ is the electric charge, $h$ is Planck's constant, and $\left\langle\lambda_{0}\right\rangle$ is an averaged EMFP over the Fermi surface area. With the electrical resistivity of $5.3 \mu \Omega \mathrm{cm}$ at $293 \mathrm{~K}$ (Ref. 27) and the Fermi surface area of $15.1 \AA^{-2}$, the EMFP of W is determined as $19.1 \mathrm{~nm}$.

TABLE II. Film identification, film thickness, deposition temperature, film resistivities at $293 \mathrm{~K}$ in the as-deposited and annealed states, film resistivities at $4.2 \mathrm{~K}$, and the residual resistivity ratio (RRR) for the subset of the annealed films. FWHM values from x-ray rocking curves for the single-crystal films are also given. The films were ex situ annealed at $850{ }^{\circ} \mathrm{C}$ for $2 \mathrm{~h}$ in $\mathrm{Ar}-4 \% \mathrm{H}_{2}$.

\begin{tabular}{|c|c|c|c|c|c|c|c|c|}
\hline $\begin{array}{l}\text { Film } \\
\text { i.d. }\end{array}$ & $\begin{array}{l}\text { Film } \\
\text { thickness (nm) }\end{array}$ & $\begin{array}{l}\text { Roughness } \\
\text { RMS (nm) }\end{array}$ & & $\begin{array}{c}\text { Resistivity at } \\
293 \mathrm{~K}(\mu \Omega \mathrm{cm})\end{array}$ & $\begin{array}{l}\text { Resistivity at } \\
4.2 \mathrm{~K}(\mu \Omega \mathrm{cm})\end{array}$ & RRR & & $\begin{array}{c}\text { FWHM } \\
\text { (degrees) }\end{array}$ \\
\hline & & Annealed & As-deposited & Annealed & Annealed & & As-deposited & Annealed \\
\hline A & 9.8 & 0.46 & 12.2 & 10.1 & 4.6 & 2.2 & 0.09 & 0.08 \\
\hline B & 19.9 & 0.31 & 9.8 & 8.6 & 3.5 & 2.5 & - & 0.09 \\
\hline $\mathrm{C}$ & 41.4 & 0.50 & 8.6 & 7.7 & 2.4 & 3.2 & 0.08 & 0.09 \\
\hline $\mathrm{D}$ & 62.8 & 0.42 & 7.8 & 7.4 & 2.2 & 3.3 & - & 0.16 \\
\hline $\mathrm{E}$ & 123.1 & 0.28 & 6.9 & 6.6 & 1.5 & 4.4 & - & 0.16 \\
\hline $\mathrm{F}$ & 187.1 & 0.69 & 6.8 & 6.4 & 1.3 & 4.8 & 0.37 & 0.23 \\
\hline $\mathrm{G}$ & 299.7 & 0.45 & 6.5 & 6.3 & 1.3 & 4.8 & - & 0.33 \\
\hline
\end{tabular}




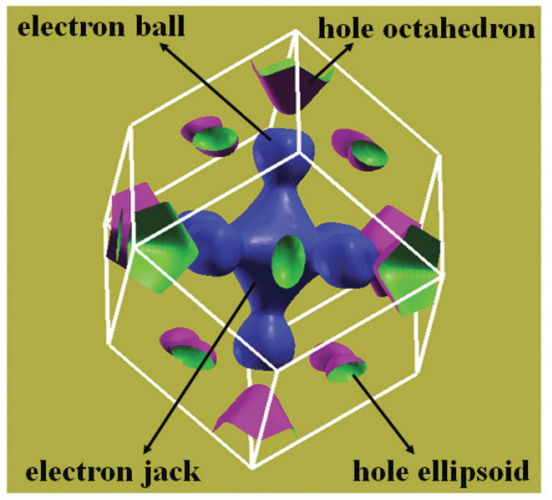

FIG. 1. (Color online) The Fermi surface of W constructed based on density functional theory (DFT) calculation. The four closed parts of the surface are labeled with their commonly used designations.

Table II gives the measured thicknesses of the $\mathrm{W}$ layers and the root mean squared (RMS) roughness of the annealed samples. Resistivities at $293 \mathrm{~K}$ for the films in the as-deposited state and after the $850{ }^{\circ} \mathrm{C}$ anneal are also given in the table. The resistivities at $4.2 \mathrm{~K}$ are given only for the annealed state. Table II also gives the residual resistivity ratio (RRR) for the annealed samples. Angular FWHMs from the rocking curves are provided for all the annealed films and for a subset of the as-deposited films.

Figure 2 presents the XRD pattern for film $\mathrm{C}$ in the annealed state. Only the $\mathrm{Al}_{2} \mathrm{O}_{3}$ substrate peaks and the $\{110\}$ peak of $\alpha$-W [body-centered cubic (bcc)] are seen in the figure. ${ }^{28}$ The inset shows the rocking curve for the $\alpha$-(110) peak for film C, where the rocking curve FWHM was $0.09^{\circ}$.

A high-resolution TEM micrograph for film $\mathrm{F}$ is shown in Fig. 3. The selected area diffraction pattern for the $\mathrm{W}$ layer, presented as the inset in the top right, evidences a [11 1 ] zone axis pattern. The measured interplanar spacing in this micrograph along the film normal is $2.20 \AA$, which is close to the expected value of $2.24 \AA$ for $d_{110}$ for $\alpha$-W. Figure 3 shows the epitaxial interface between the $(11 \overline{2} 0) \mathrm{Al}_{2} \mathrm{O}_{3}$ substrate and the (110) W film, which is further evidenced by singlecrystal diffraction patterns of the Fourier-transformed lattice images in the three different regions of Fig. 3 presented as insets on the left.

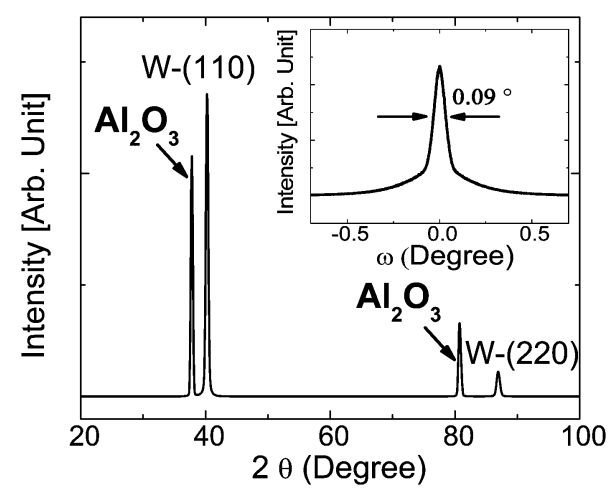

FIG. 2. $\theta-2 \theta$ XRD patterns for film $\mathrm{C}$ in the annealed state. The substrate peaks from left to right are $11 \overline{2} 0$ and $22 \overline{4} 0$, respectively. The inset shows the rocking curve $(\omega$ scan) for the $\mathrm{W}-110$ peak. The FWHM value for this film was $0.09^{\circ}$ (See Table II.)

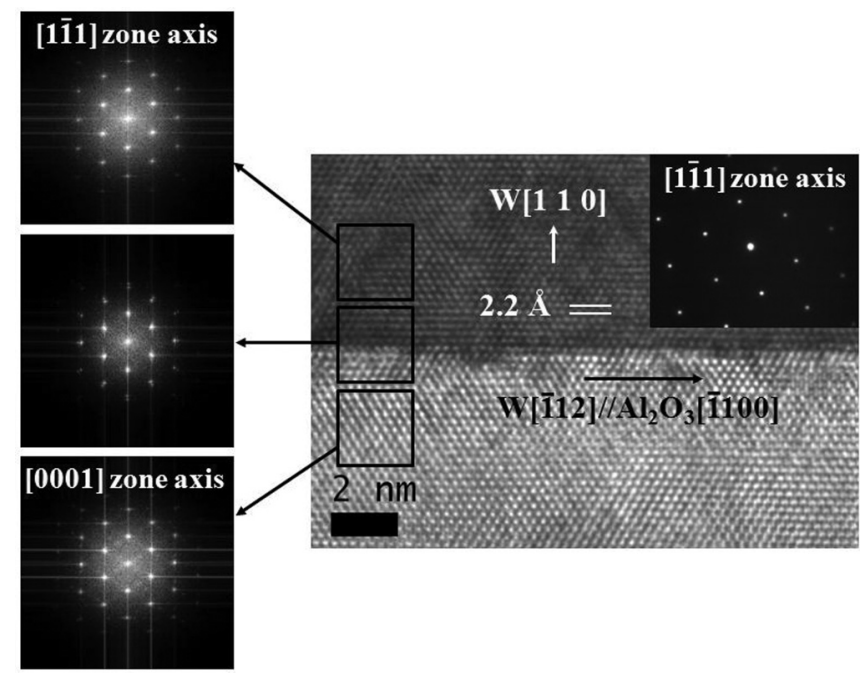

FIG. 3. High-resolution transmission electron micrograph of film $\mathrm{F}$ in the annealed state. The selected area diffraction pattern for the $\mathrm{W}$ layer is presented as the inset in the top right corner of the HRTEM micrograph. Fourier transforms of the lattice images in the three different regions are presented as insets on the left.

The FWHMs of the XRD rocking curves were used to estimate the dislocation density in the films using the relationship ${ }^{29,30}$

$$
D_{\text {dislocation }} \sim \frac{\mathrm{FWHM}^{2}}{9 b^{2}},
$$

where $D_{\text {dislocation }}$ is the dislocation density in the film and $b$ is the length of the Burgers vector. With the measured FWHM values and the Burgers vector of $1 / 2\left[\begin{array}{lll}1 & 1 & 1\end{array}\right](=0.274 \mathrm{~nm}),{ }^{31}$ the estimated dislocation densities for the annealed films are in the range of $2.9 \times 10^{8}-4.9 \times 10^{9} \mathrm{~cm}^{-2}$. This level of dislocation density is not expected to significantly contribute to the electrical resistivity of the films since epitaxial $\mathrm{W}$ films with higher rocking curve FWHM values than seen here exhibited resistivities close to the known bulk resistivity. ${ }^{32}$

The resistivity data at 293 and $4.2 \mathrm{~K}$ given in Table II are plotted in Fig. 4. The data were fitted to the FS model using Eq. (1), with the surface specularity $p$ treated as a

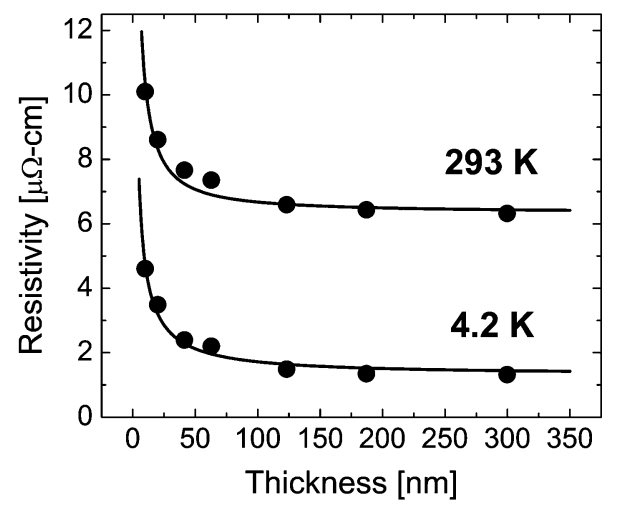

FIG. 4. Resistivities of annealed, epitaxial single-crystal (110) W films at 293 and $4.2 \mathrm{~K}$ are plotted as a function of film thickness. The data points correspond to films A-G in Table II. The solid curves are the best fit curves to the Fuchs-Sondheimer (FS) model. 


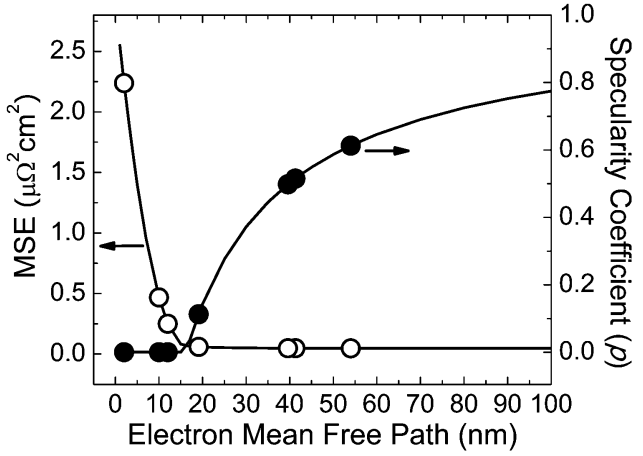

FIG. 5. Specularity parameter $p$ and the associated mean square error are plotted vs the EMFP. The discrete data points represent the EMFPs given in Table I and the DFT-calculated EMFP.

fitting parameter. In using Eq. (1) account had to be taken of the fact that the purity of the $\mathrm{W}$ sputtering target was only $99.95 \%$, and thus impurities clearly contributed to film resistivity. To account for their contribution, the resistivity of the 300-nm-thick film was taken as the bulk resistivity at the two temperatures. The EMFP of the films at these temperatures was then calculated by taking the product $\rho_{\mathrm{o}}\langle\lambda\rangle_{\mathrm{o}}=1.01 \times$ $10^{-15} \Omega \mathrm{m}^{2}$ to be independent of temperature.

From the fits, the specularity parameter is found to be $p=$ $0.11 \pm 0.082$ with the mean square error (MSE) of 0.06 , the error limits selected so as to give a $60 \%$ increase of the MSE. This low value of $p$ indicates nearly fully diffuse scattering from the surfaces, as has also been seen for epitaxial $\mathrm{Cu}$ films. ${ }^{33,34}$ The value of MSE for the fits is significantly lower than the MSE of 0.21 found in the work by Mikhailov et al. where an EMFP of $39.6 \mathrm{~nm}$ was used for the fits ${ }^{9}$ (see Table I). Furthermore, the fits to the resistivity data reported here did not require the use of an ad hoc parameter such as a dead layer, as was done by Mikhailov et al.. ${ }^{9}$

Although the FS model with the EMFP of $19.1 \mathrm{~nm}$ and $p=0.11$ provides a good description of the resistivity data of $\mathrm{W}$ films prepared here, it is useful to determine whether this parameter set represents a unique pair. To this end, the specularity parameter $p$ and the associated MSE were found by fitting the resistivity data of the films as the EMFP was increased from 1 to $500 \mathrm{~nm}$. In Fig. 5, the values of $p$ and MSE are plotted for EMFP values up to $100 \mathrm{~nm}$ and shown as a solid curve. The discrete data points in the figure are for the EMFPs given in Table I and the EMFP obtained by DFT calculation $(19.1 \mathrm{~nm})$, and it should be noted that all the prior conflicting values of $p$ from Table I fall on this single $p$ vs EMFP curve. Further, it can be seen that the MSE of the fitting shows a very sharp decrease with increasing EMFP, reaching its low plateau for an EMFP near the DFT value of $19.1 \mathrm{~nm}$. The specularity parameter $(p)$ shows a complementary increase and reaches a value of 0.61 at an EMFP of $54 \mathrm{~nm}$ (and 0.95 at an EMFP of $500 \mathrm{~nm}$, not shown). Clearly, the use of the FS model does not provide a physically meaningful upper bound to the EMFP. However, using the $F$-test to examine the error values (i.e., the set of differences between the calculated resistivities and the experimental ones) does allow a lower bound to be placed on the EMFP of $12 \mathrm{~nm}$ at a confidence level of $>93 \%$ and $10 \mathrm{~nm}$ at a confidence level of $>99 \%$. Thus, the measured film resistivities clearly render the values of EMFP by Rossnagel et al. ${ }^{10}$ and Choi et al. ${ }^{4}$ in Table I unlikely, but are not able to select among the other entries in the table. The difficulty in establishing an upper-bound EMFP using the FS model is inherently associated with the nature of the specularity coefficient defined in the model; i.e., even with a very long EMFP, the fitting can be successful with $p$ falsely becoming very close to 1 (almost mirrorlike reflection at surfaces). Therefore, the approach of estimating an EMFP as a result of fitting resistivity data to the FS model, for example, in the work of Ref. 7 in Table I, can result in a significantly longer value than the actual EMFP.

In order to determine at what linewidth the resistivity of polycrystalline $\mathrm{W}$ lines will cross below that of $\mathrm{Cu}$, it will be necessary to determine the grain boundary reflection coefficient for W. This is the subject of ongoing work and will be reported in future publications.

\section{CONCLUSION}

Using the Fermi surface area calculated using the density functional theory (DFT), the electron mean free path (EMFP) of pure, bulk W due to phonon scattering at room temperature was determined as $19.1 \mathrm{~nm}$. The Fuchs-Sondheimer (FS) model with this value of EMFP and nearly fully diffuse surface scattering was then shown to provide a very good description of the resistivity as a function of thickness for (110) oriented epitaxial $\mathrm{W}$ films deposited on (1120) $\mathrm{Al}_{2} \mathrm{O}_{3}$ substrates. The resistivity data and the $\mathrm{FS}$ model allowed a lower bound on the EMFP to be placed at $12 \mathrm{~nm}$ at a confidence level of $>93 \%$ and $10 \mathrm{~nm}$ at a confidence level of $>99 \%$, whereas no upper bound to the EMFP can be established using the FS model.

\section{ACKNOWLEDGMENTS}

Financial support from the Alessandro and Piermaria Reggiori Fellowship, the Bertucci Graduate Fellowship, the Department of Materials Science and Engineering, Intel Corporation, and SRC Tasks No. 1292.008 and No. 2121.001 are acknowledged. Portions of this research were carried out at the Stanford Synchrotron Radiation Lightsource, a Directorate of SLAC National Accelerator Laboratory and an Office of Science User Facility operated for the US Department of Energy Office of Science by Stanford University. D.N. would like to acknowledge the National Science Foundation computational resources provided through the TeraGrid at the Texas Advanced Computing Center under Grant No. TGDMR120002. D.N. would also like to acknowledge Daniel Siderius who shares a MATLAB code online for calculating an isosurface area for a three-dimensional object.
${ }^{1}$ J. R. Lloyd, J. Clemens, and R. Snede, Microelectron. Reliab. 39, 1595 (1999).
${ }^{2}$ J. R. Lloyd, M. W. Lane, E. G. Liniger, C.-K. Hu, T. M. Shaw, and R. Rosenberg, IEEE Trans. Device Mater. Reliab. 5, 113 (2005). 
${ }^{3}$ C. S. Hau-Riege, Microelectron. Reliab. 44, 195 (2004).

${ }^{4}$ D. Choi, B. Wang, S. Chung, X. Liu, A. Darbal, A. Wise, N. T. Nuhfer, K. Barmak, A. Warren, K. R. Coffey, and M. F. Toney, J. Vac. Sci. Technol. A 29, 051512 (2011).

${ }^{5}$ T. Sun, B. Yao, A. P. Warren, K. Barmak, M. F. Toney, R. E. Peale, and K. R. Coffey, Phys. Rev. B 81, 155454 (2010).

${ }^{6}$ D. Josell, S. H. Brongersma, and Z. Tökei, Annu. Rev. Mater. Res. 39, 231 (2009).

${ }^{7}$ W. Steinhögl, G. Steinlesberger, M. Perrin, G. Scheinbacher, G. Schindler, M. Traving, and M. Engelhardt, Microelectron. Eng. 82, 266 (2005).

${ }^{8}$ A. J. Learn and D. W. Foster, J. Appl. Phys. 58, 2001 (1985).

${ }^{9}$ G. M. Mikhailov, A. V. Chernykh, and V. T. Petrashov, J. Appl. Phys. 80, 948 (1996).

${ }^{10}$ S. M. Rossnagel, I. C. Noyan, and C. Cabral Jr., J. Vac. Sci. Technol. B 20, 2047 (2002).

${ }^{11}$ K. Fuchs, Proc. Cambridge Philos. Soc. 34, 100 (1938).

${ }^{12}$ E. H. Sondheimer, Adv. Phys. 1, 1 (1952).

${ }^{13}$ A. F. Mayadas and M. Shatzkes, Phys. Rev. B 1, 1382 (1970).

${ }^{14}$ D. Choi, Ph.D. thesis, Carnegie Mellon University, Pittsburgh, PA, 2011.

${ }^{15}$ J. P. Perdew, K. Burke, and M. Ernzerhof, Phys. Rev. Lett. 77, 3865 (1996)

${ }^{16}$ X. Gonze, B. Amadon, P.-M. Anglade, J.-M. Beuken, F. Bottin, P. Boulanger, F. Bruneval, D. Caliste, R. Caracas, M. Côté, T. Deutsch, L. Genovese, Ph. Ghosez, M. Giantomassi, S. Goedecker, D. R. Hamann, P. Hermet, F. Jollet, G. Jomard, S. Leroux, M. Mancini, S. Mazevet, M. J. T. Oliveira, G. Onida, Y. Pouillon, T. Rangel, G.-M. Rignanese, D. Sangalli, R. Shaltaf, M. Torrent, M. J. Verstraete, G. Zerah, and J. W. Zwanziger, Comput. Phys. Commun. 180, 2582 (2009).
${ }^{17}$ C. Hartwigsen, S. Goedecker, and J. Hutter, Phys. Rev. B 58, 3641 (1998).

${ }^{18}$ A. Kokalj, Comput. Mater. Sci. 28, 155 (2003).

${ }^{19} \mathrm{~J}$. Lindblat and I. Nyström, Proceedings of the 10th International Conference on Discrete Geometry for Computer Imagery (SpringerVerlag, London, 2002), pp. 267-278.

${ }^{20}$ M. Yoshimoto, T. Maeda, T. Ohnishi, H. Koinuma, O. Ishiyama, M. Shinohara, M. Kubo, R. Miura, and A. Miyamoto, Appl. Phys. Lett. 67, 2615 (1995).

${ }^{21}$ A. R. Wildes, J. Mayer, and K. Theis-Bröhl, Thin Solid Films 401, 7 (2001).

${ }^{22}$ L. J. van der Pauw, Philips Res. Rep. 13, 1 (1958).

${ }^{23}$ D. M. Sparlin and J. A. Marcus, Phys. Rev. 144, 484 (1966).

${ }^{24}$ R. F. Girvan, A. V. Gold, and R. A. Phillips, J. Phys. Chem. Solids 29, 1485 (1968).

${ }^{25}$ E. Fawcett and D. Griffiths, J. Phys. Chem. Solids, 23, 1631 (1962).

${ }^{26}$ A. B. Pippard, Physical Society 33, 176 (1960).

${ }^{27}$ CRC Handbook of Chemistry and Physics, 92th ed. (Taylor \& Francis, London, 2012), p. 12-41 [http://www.hbcpnetbase.com/].

${ }^{28}$ N. Cherief, D. Givord, A. Liénard, K. Mackay, O. F. K. McGrath, J. P. Rebouillat, F. Robaut, and Y. Souche, J. Magn. Magn. Mater. 121, 94 (1993).

${ }^{29}$ X. Q. Shen, H. Matsuhata, and H. Okumura, Appl. Phys. Lett. 86, 021912 (2005).

${ }^{30}$ P. Gay, P. B. Hirsch, and A. Kelly, Acta Metall. 1, 316 (1953).

${ }^{31}$ E. J. Grier, M. L. Jenkins, A. K. Petford-Long, R. C. C. Ward, and M. R. Wells, Thin Solid Films 358, 94 (2000).

${ }^{32}$ J. H. Souk, A. Segmuller, and J. Angilello, J. Appl. Phys. 62, 509 (1987).

${ }^{33}$ J. M. Purswani and D. Gall, Thin Solid Films 516, 465 (2007).

${ }^{34}$ E. T. Krastev, L. D. Voice, and R. G. Tobin, J. Appl. Phys. 79, 6865 (1996). 